\title{
Classification of the wine sites "world heritage Unesco": Situational analysis and prospects / Classement des sites viticoles « Patrimoine mondial Unesco», état des lieux et perspectives
}

\author{
Joël Rochard \\ IFV (Institut Français de la Vigne et du Vin), 17 rue Jean Chandon Moët, 51200 Epernay, France
}

\begin{abstract}
The inheritance is the legacy of the past from which we profit today and who we transmit to the generations to come. UNESCO adopted in 1972 a convention concerning heritage protection world, cultural and natural. The cultural heritage relates to the monuments, whole of constructions and goods with historical values, esthetics, archaeological, scientific, ethnological or anthropological. The natural heritage relates to the formations physical, biological and geological remarkable, the surfaces of an exceptional value of the point of the sight of science, conservation or natural beauty and the habitats of threatened animal species and vegetable. The wine landscapes are generally recognized among the most remarkable forms of landscapes resulting from the human activity, at the same time by the mark which they print with the territory and by the cultural traditions which theirs are associated, which leads to the classification of fifteen European wine sites. This approach is integrated in a vision of patrimonial governance of the wine terroir which associate the orientations of the international organization of the vine and the wine opposite in particular of the concept of terroir and Viticulture. The objective of the communication is to establish an inventory of fixtures of the classified as wine sites "World heritage UNESCO" and to put in prospect the patrimonial approach for the world wine sector.
\end{abstract}

Résumé. Le patrimoine est l'héritage du passé dont nous profitons aujourd'hui et que nous transmettons aux générations à venir. L'Unesco a adopté en 1972 une convention concernant la protection du patrimoine mondial, culturel et naturel. Le patrimoine culturel concerne les monuments, ensembles de constructions et biens avec des valeurs historiques, esthétiques, archéologiques, scientifiques, ethnologiques ou anthropologiques. Le patrimoine naturel concerne les formations physiques, biologiques et géologiques remarquables, les aires d'une valeur exceptionnelle du point du vue de la science, de la conservation ou de la beauté naturelle et les habitats d'espèces animales et végétales menacées. Les paysages de vigne sont généralement reconnus parmi les formes les plus remarquables de paysages résultant de l'activité humaine, à la fois par la marque qu'ils impriment au territoire et par les traditions culturelles qui leurs sont associés, ce qui aboutit au classement d'une quinzaine de sites viticoles européens. Cette approche s'intègre dans une vision de gouvernance patrimoniale des terroirs viticoles qui associent les orientations de l'organisation internationale de la vigne et du vin vis-à-vis notamment du concept de terroir et de Vitiviniculture durable. L'objectif de la communication est d'établir un état des lieux des sites viticoles classés « Patrimoine mondial Unesco » et de mettre en perspective l'approche patrimoniale pour le secteur viticole mondial.

\section{Introduction}

L'œnotourisme prend une part grandissante dans la valorisation des territoires viticoles. C'est également un facteur qui participe à l'image et à la valeur ajoutée des vins de ces régions. Parallèlement à l'attrait culturel pour la vigne, le vin et la dégustation, « l'EEnotourisme » est souvent motivé par une approche écologique et patrimoniale des caves et des terroirs. Ainsi émerge le concept « d'écoœnotourisme » qui associe notamment, en liaison avec le développement durable, les paysages, la biodiversité, ainsi que l'éco-conception des caves.

Parallèlement les viticulteurs ont intérêt à offrir un paysage de qualité, support emblématique de l'activité touristique de leur région, vecteur d'image de leur métier et de leurs produits. A propos de la valeur symbolique, émotionnelle, affective du terroir, S. Michel précise que «Nous sommes ce que nous mangeons. La méconnaissance de ce que nous avons dans notre assiette ou notre verre conduit à une perte d'identité, mais heureusement le terroir restaure le lien entre le consommateur et l'aliment. Les valeurs symboliques de l'aliment sont celles du terroir mais attention, toute contamination du terroir ; qu'elle soit physique, chimique, biologique ou visuelle, contamine le produit dans l'esprit $d u$ client $\gg$. Cette réflexion souligne que le terroir, et dans son prolongement la cave, sont des atouts, supports de valorisation, mais à l'inverse une dénaturation des paysages, ou une approche peu valorisante de la cave, peut dégrader la perception du terroir et indirectement l'image du vin et de la filière. 


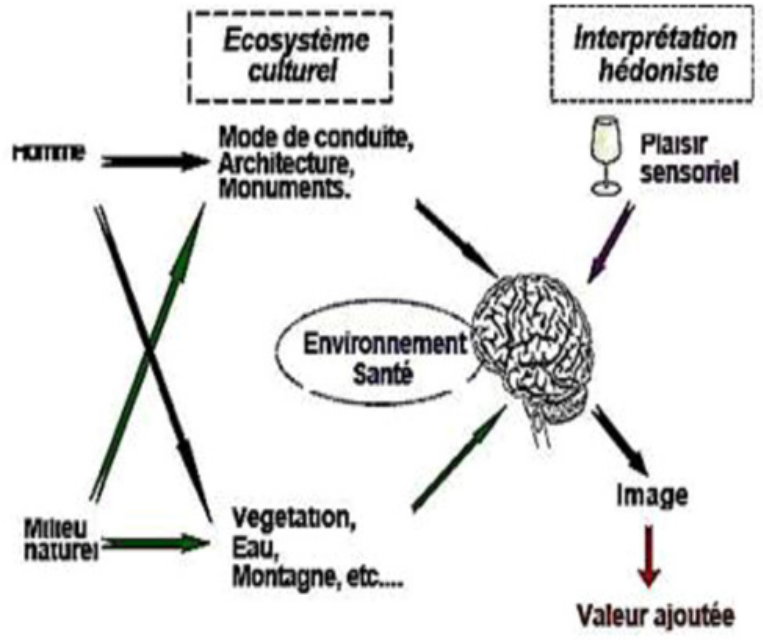

Figure 1. Composante de l'image d'un vin (Source : J. Rochard, Traité de Viticulture et d'œnologie durable, Editions Avenir Enologie, 2005) [3].

La Convention européenne du paysage a défini en 2000 le paysage comme « une partie du territoire telle que perçue par les populations, dont le caractère résulte de l'action de facteurs naturels et / ou humains et de leurs interrelations »[1]. Au-delà de l'approche purement descriptive, le paysage est l'expression esthétique de l'écosystème culturel que constitue le terroir, patrimoine naturel domestiqué et valorisé par l'homme. Cette dimension, qui relie la terre à l'esprit, associe en premier lieu le savoir-faire technique du vigneron, à la fois architecte et jardinier, au sens noble du terme, du terroir. Ce savoir-faire est le fruit à la fois d'observations empiriques, acquises de génération en génération, et d'une recherche permanente associée notamment au mode de conduite de la vigne, à l'aménagement, à la gestion des sols et des coteaux. Parallèlement à la dimension purement viticole, les paysages s'enrichissent très souvent d'autres composantes, esthétiques en liaison avec le milieu naturel, l'architecture des caves et des villages viticoles (Fig. 1) [2].

Cependant, le paysage viticole est parfois menacé. En premier lieu, l'arrachage, l'abandon ou l'urbanisation constituent des risques définitifs de disparition de panoramas remarquables. Des sources d'agressions complémentaires peuvent également dénaturer ces paysages (lignes électriques, aménagements routiers, éoliennes, panneaux publicitaires) (Fig. 2) [3].

La filière viticole est parfois également à l'origine d'une dégradation esthétique des paysages. La restructuration des coteaux (suppression des murets), la mise en place d'un mode de conduite atypique par rapport aux pratiques traditionnelles, le choix de piquets inesthétiques, sont autant d'exemples, de nature à porter préjudice à la dimension paysagère d'un terroir viticole. Parallèlement, la construction des chais, au-delà des aspects strictement fonctionnels, s'inscrit très souvent dans une démarche originale d'architecture, expression d'une culture voire d'une philosophie, destinée à exprimer auprès du public une singularité, l'image d'un vigneron, négociant ou groupe de producteurs. Certaines caves ; traditionnelles ou modernes, sont de véritables œuvres d'art qui s'intègrent dans les valeurs patrimoniales du territoire.

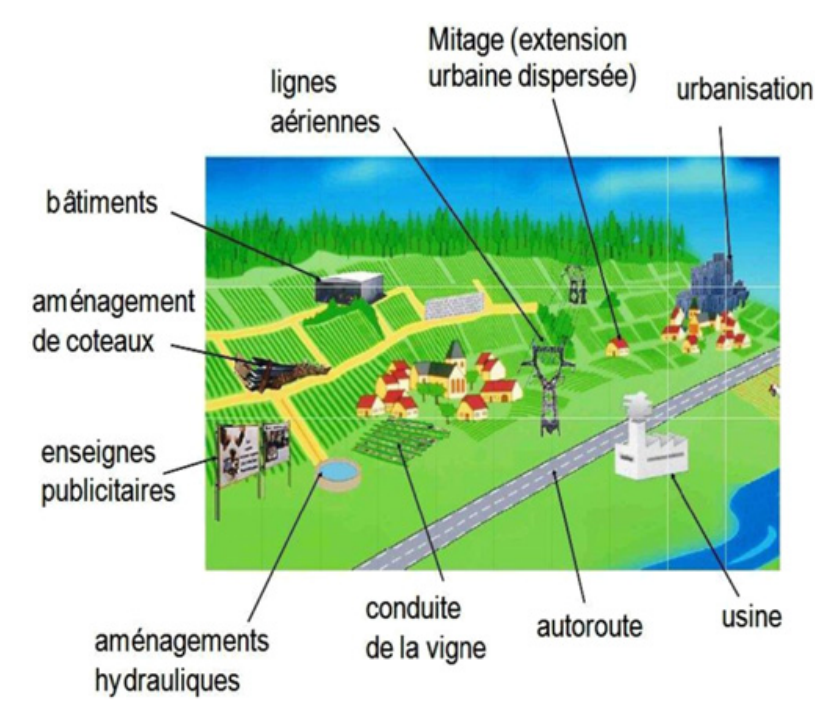

Figure 2. Sources d'agression des paysages viticoles (Source : J. Rochard, Traité de Viticulture et d'œnologie durable, Editions Avenir (Enologie, 2005).

Il est primordial d'éviter que le paysage soit un simple sous-produit des pratiques viticoles et territoriales, ce qui justifie de développer localement une « gouvernance patrimoniale » qui associe l'ensemble des acteurs du territoire (organisations viticoles, collectivités territoriales, office du tourisme et experts locaux) dans une approche pluridisciplinaire. La motivation des acteurs locaux pour s'intégrer dans une dynamique collective est souvent liée à un 《retour image » pour le produit et la région associée à un processus de labellisation.

« Nos patrimoines culturels et naturels sont deux sources irremplaçables de vie et d'inspiration. [...] Ce qui rend exceptionnel le concept de patrimoine mondial est son application universelle. Les sites du patrimoine mondial appartiennent à tous les peuples du monde, sans tenir compte du territoire sur lequel ils sont situés. » Source Unesco, centre du patrimoine mondial [4].

L'Unesco, initialement créée pour « contribuer au maintien de la paix et de la sécurité en resserrant, par l'éducation, la science et la culture, la collaboration entre nations » s'est notamment impliqué pour conserver les biens dits culturels ou naturels d'importance pour l'héritage commun de l'humanité. Après avoir entrepris en 1964 les travaux de sauvetage des temples d'Abou Simbel contre la montée des eaux du lac Nasser, à la suite de la construction du Haut barrage d'Assouan en Égypte, l' Unesco a établi en 1972 une convention pour la protection du patrimoine mondial, culturel et naturel. Cette démarche s'est traduite en 1976 par la création du comité patrimoine mondial qui a pour mission de faire connaître et de protéger les sites que l'organisation considère comme exceptionnels avec la mise en place de critères de sélection Pour ce faire, et dans un souci d'objectivité, ont été mis en place des critères.

Scène de découpe des colosses de la façade du grand temple d'Abou Simbel lors du sauvetage de deux temples en 1967.

Dans les années 1960, alors que le président égyptien Gamal Abdel Nasser fait construire le haut barrage d'Assouan sur le Nil afin de produire de l'électricité, 
d'augmenter les surface cultivables et d'éliminer la crue du Nil en aval du barrage, l'Unesco entreprend de sauver ces deux monuments majeurs du patrimoine mondial, de la montée des eaux du lac Nasser. Ce chef-d'œuvre nubien de Ramsès II, situé à l'origine sur les collines sacrées de Méha et d'Ibshek, a été démonté entièrement et reconstruit plus haut, au bord du lac, sur une colline artificielle à l'abri de la montée des eaux. Cette approche de l'Unesco a initié la notion de patrimoine culturel, naturel puis immatériel qui a progressivement associé le secteur viticole (Fig. 3).

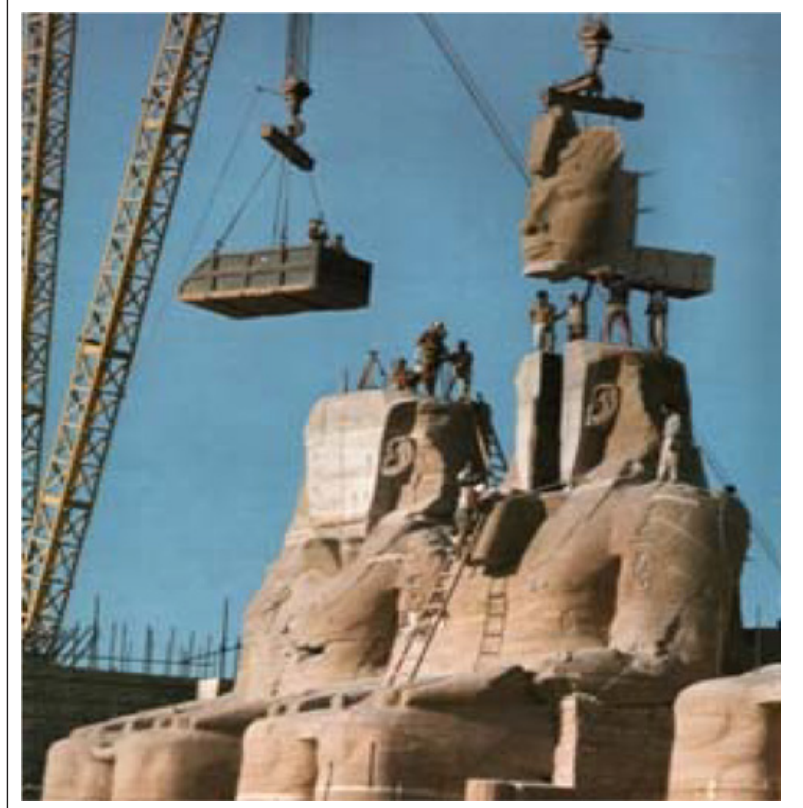

Figure 3. Découpe des colosses de la façade du grand temple d'Abou Simbel. Photo Per-Olow Anderson/wikipédia, 1967.

« Le patrimoine est l'héritage du passé, dont nous profitons aujourd'hui et que nous transmettons aux générations à venir. 》 Extrait de la Convention de 1972 de l'Unesco pour la protection du patrimoine mondial, culturel et naturel [4].

\section{Présentation du patrimoine mondial Unesco}

Le patrimoine mondial, ou patrimoine de l'humanité, désigne un ensemble de biens qui présentent une valeur universelle exceptionnelle justifiant leur inscription sur une liste établie par le comité du patrimoine mondial de l'Organisation des Nations Unies pour l'Education, la Science et la Culture (UNESCO).

Les principales motivations de la convention pour la protection du patrimoine mondial culturel et naturel (Unesco 1972).

- Constatant que le patrimoine culturel et le patrimoine naturel sont de plus en plus menacés de destruction non seulement par les causes traditionnelles de dégradation mais encore par l'évolution de la vie sociale et économique qui les aggrave par des phénomènes d'altération ou de destruction encore plus redoutables,
- Considérant que la dégradation ou la disparition d'un bien du patrimoine culturel et naturel constitue un appauvrissement néfaste du patrimoine de tous les peuples du monde,

- Considérant que la protection de ce patrimoine à l'échelon national reste souvent incomplète en raison de l'ampleur des moyens qu'elle nécessite et de l'insuffisance des ressources économiques, scientifiques et techniques du pays sur le territoire duquel se trouve le bien à sauvegarder,

- Considérant que les conventions, recommandations et résolutions internationales existantes en faveur des biens culturels et naturels démontrent l'importance que présente, pour tous les peuples du monde, la sauvegarde de ces biens uniques et irremplaçables à quelque peuple qu'ils appartiennent,

- Considérant que certains biens du patrimoine culturel et naturel présentent un intérêt exceptionnel qui nécessite leur préservation en tant qu'élément du patrimoine mondial de l'humanité tout entière,

- Considérant que devant l'ampleur et la gravité des dangers nouveaux qui les menacent il incombe à la collectivité internationale tout entière de participer à la protection du patrimoine culturel et naturel de valeur universelle exceptionnelle, par l'octroi d'une assistance collective qui sans se substituer à l'action de l'Etat intéressé la complétera efficacement,

- Considérant qu'il est indispensable d'adopter à cet effet de nouvelles dispositions conventionnelles établissant un système efficace de protection collective du patrimoine culturel et naturel de valeur universelle exceptionnelle.

L'objectif principal de la liste du patrimoine mondial est de faire connaître et de protéger les sites que l'organisation considère comme exceptionnels. Pour ce faire, et dans un souci d'objectivité, ont été mis en place une série de critères [5]. À l'origine, seuls existaient les sites culturels (1978), dont l'inscription sur la liste était régie par six critères. Puis, à la suite notamment d'un souci de rééquilibrage du patrimoine mondial entre les continents, sont apparus les sites naturels et quatre nouveaux critères.

En 2005, tous les critères ont été fondus en 10 critères uniques applicables à tous les sites' :

- Représenter un chef-d'œuvre du génie créateur humain.

- Témoigner d'un échange d'influences considérable pendant une période donnée ou dans une aire culturelle déterminée, sur le développement de l'architecture ou de la technologie, des arts monumentaux, de la planification des villes ou de la création de paysages.

- Apporter un témoignage unique ou du moins exceptionnel sur une tradition culturelle ou une civilisation vivante ou disparue.

- Offrir un exemple éminent d'un type de construction ou d'ensemble architectural ou technologique ou de paysage illustrant une ou des périodes significative(s) de l'histoire humaine.

- Être un exemple éminent d'établissement humain traditionnel, de l'utilisation traditionnelle du territoire ou de la mer. 
- Être directement ou matériellement associé à des événements ou des traditions vivantes, des idées, des croyances ou des œuvres artistiques et littéraires ayant une signification universelle exceptionnelle.

- Représenter des phénomènes naturels ou des aires d'une beauté naturelle et d'une importance esthétique exceptionnelles.

- Être des exemples éminemment représentatifs des grands stades de l'histoire de la Terre.

- Être des exemples éminemment représentatifs de processus écologiques et biologiques en cours dans l'évolution et le développement des écosystèmes.

- Contenir les habitats naturels les plus représentatifs et les plus importants pour la conservation in situ de la diversité biologique.

Parallèlement en 2003, l'Unesco a également établi une convention sur la sauvegarde du patrimoine immatériel. «Le patrimoine culturel immatériel ne comprend pas seulement les traditions héritées du passé, mais aussi les pratiques rurales et urbaines contemporaines, propres à divers groupes culturels. On entend par «patrimoine culturel immatériel » les pratiques, représentations, expressions, connaissances et savoir-faire - ainsi que les instruments, objets, artefacts et espaces culturels qui leur sont associés - que les communautés, les groupes et, le cas échéant, les individus reconnaissent comme faisant partie de leur patrimoine culturel. Ce patrimoine culturel immatériel, transmis de génération en génération, est recréé en permanence par les communautés et groupes en fonction de leur milieu, de leur interaction avec la nature et de leur histoire, et leur procure un sentiment d'identité et de continuité, contribuant ainsi à promouvoir le respect de la diversité culturelle et la créativité humaine » (UNESCO 2003).

L'importance du patrimoine culturel immatériel ne réside pas uniquement dans la manifestation culturelle elle-même mais également dans la richesse des connaissances et du savoir-faire qu'il transmet d'une génération à une autre. Cette transmission du savoir a une valeur sociale et économique pertinente pour les groupes minoritaires comme pour les groupes sociaux majoritaires à l'intérieur d'un État et s'intègre dans une vision de développement durable.

«Nous pouvons partager des expressions culturelles qui ont été transmises de génération en génération, ont évolué en réponse à leur environnement et contribuent à nous procurer un sentiment d'identité et de continuité...» Source UNESCO, centre du patrimoine mondial.

La demande d'inscription d'un site sur la liste du patrimoine mondial doit provenir du pays lui-même à partir d'une liste indicative issue d'un inventaire des biens que chaque Etat a l'intention de proposer pour inscription au cours des années à venir. La demande doit inclure un plan exposant en détail la gestion et la protection.

Le comité du patrimoine mondial se réunit une fois par an et étudie les propositions d'inscription à partir d'évaluations techniques. Ces évaluations indépendantes des sites culturels et naturels proposés sont réalisées par deux organismes consultatifs, le Conseil international des monuments et des sites (ICOMOS) et l'union mondiale pour la nature (UICN) (Fig. 4).

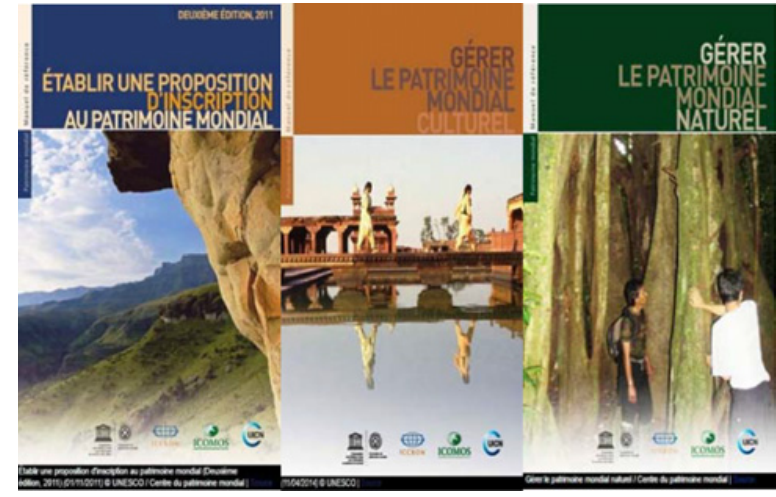

Figure 4. Plaquettes de référence de l'Unesco (http://whc. unesco.org) [6-8].

Établir une proposition d'inscription sur la Liste du patrimoine mondial est une entreprise qui demande du temps et des efforts. Il faut compter en général au moins deux ans de travail parfois beaucoup plus. Être bien préparé et bien organisé permet de procéder aussi vite et aussi aisément que possible. Les études thématiques, lorsqu'elles existent, sont une très importante source d'information propre à faciliter l'établissement d'une proposition d'inscription. Par ailleurs, la Convention fait obligation aux États de présenter au Comité du patrimoine mondial des rapports périodiques sur l'état de conservation de leurs biens inscrits sur la Liste du patrimoine mondial.

Les différents sites classés « Patrimoine Mondial » par l'Unesco intègrent la notion de « paysage essentiellement évolutif ». Ce type de paysage résulte d'une exigence à l'origine sociale, économique, administrative et/ou religieuse et a atteint sa forme actuelle par association et en réponse à son environnement naturel. C'est un paysage vivant qui conserve un rôle social actif dans la société contemporaine étroitement associé au mode de vie traditionnel dans lequel le processus évolutif continue ; en même temps, il montre des preuves manifestes de son évolution au cours des temps. Une présentation synthétique des principaux sites viticoles figure ci-dessous. Elle a été établie à partir du site Internet de l'Unesco http://whc.unesco.org/fr/list/ qui détaille les caractéristiques et les motivations du classement de chacune des régions. D'autres sites classés, non répertoriés dans cet article, peuvent également intégrer, pour une petite partie leur territoire, une zone viticole.

\section{Suisse}

Vignoble en terrasses de Lavaux en Suisse (Fig. 5)

Ce paysage s'étend sur environ $30 \mathrm{~km}$ le long du versant orienté au sud des berges du lac Léman, du château de Chillon, au cœur du canton de Vaud. Les étroites terrasses, soutenues par des murs en pierre, couvrent le bas des pentes fortement inclinées entre les villages et le lac. Bien qu'il y ait des preuves que les vignes ont commencé à être cultivées dans les environs au temps des Romains, les vignobles en terrasses actuels remontent au Xe siècle, quand les monastères bénédictins et cisterciens contrôlaient la région. Le site est un exemple exceptionnel de l'interaction pluriséculaire entre les hommes et leur environnement. 


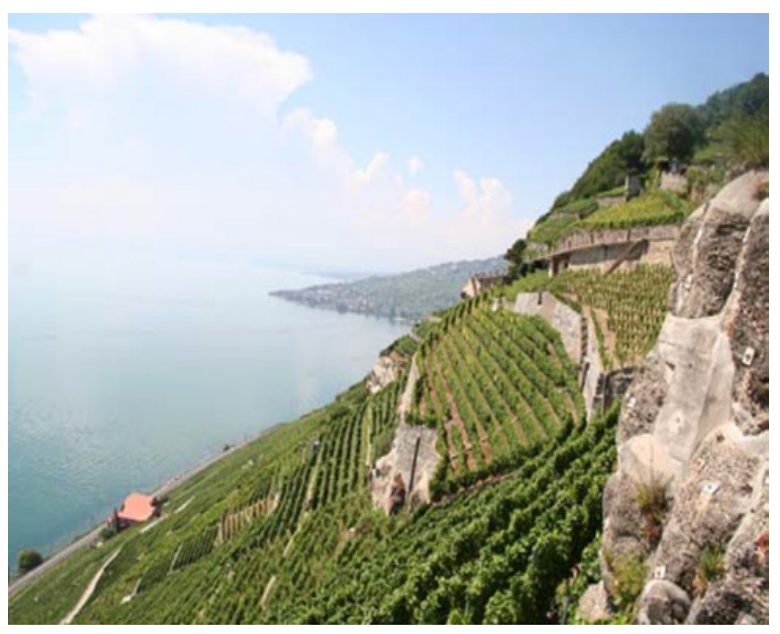

Figure 5. Paysage de Lavaux en Suisse.

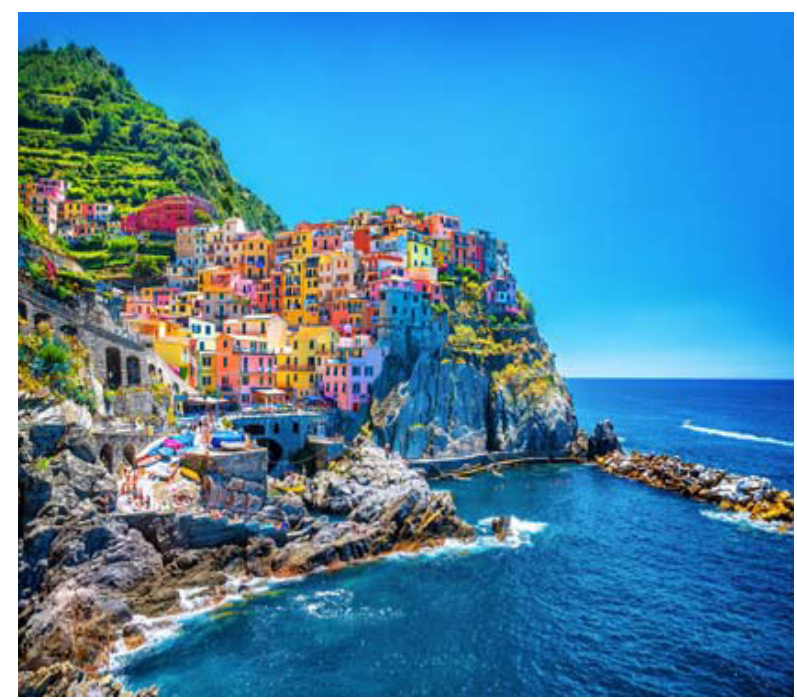

Figure 6. Cinque Terre en Italie (Photo Anna Omelchenko/ Fotolia).

\section{Italie}

Paysage de Cinque Terre en Italie (Fig. 6)

Ce territoire côtier ligurien, qui s'étend des Cinque Terre à Portovenere avec les îles Palmari Tino et Tinetto, est un paysage culturel de grande valeur panoramique et culturelle. La forme et la disposition des petites villes et le modèle du paysage environnant avec quelques vignobles, surmontant les contraintes d'un terrain escarpé et irrégulier, marquent les jalons d'une occupation humaine continue dans cette région au cours du dernier millénaire.

Vignoble italien du Piémont : Langhe-Roero et Monferrato (Fig. 7)

Le paysage classé du piémont correspond à cinq vignobles distincts et au château de Cavour, dont le nom est emblématique tant du développement du vignoble que de l'histoire de l'Italie. Situé au sud du Piémont, entre le Pô et les Apennins de Ligurie, ce paysage culturel réunit l'ensemble des processus techniques et économiques liés aux vignobles et à l'élaboration du vin, une activité caractéristique de cette région depuis des siècles. Les vignobles de Langhe-Roero et Monferrato présentent un

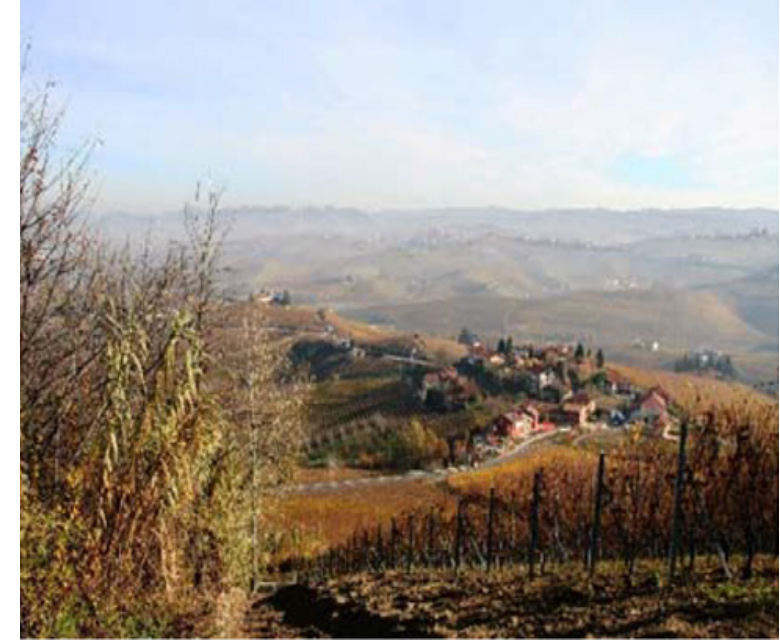

Figure 7. Paysage du Piémont en Italie.

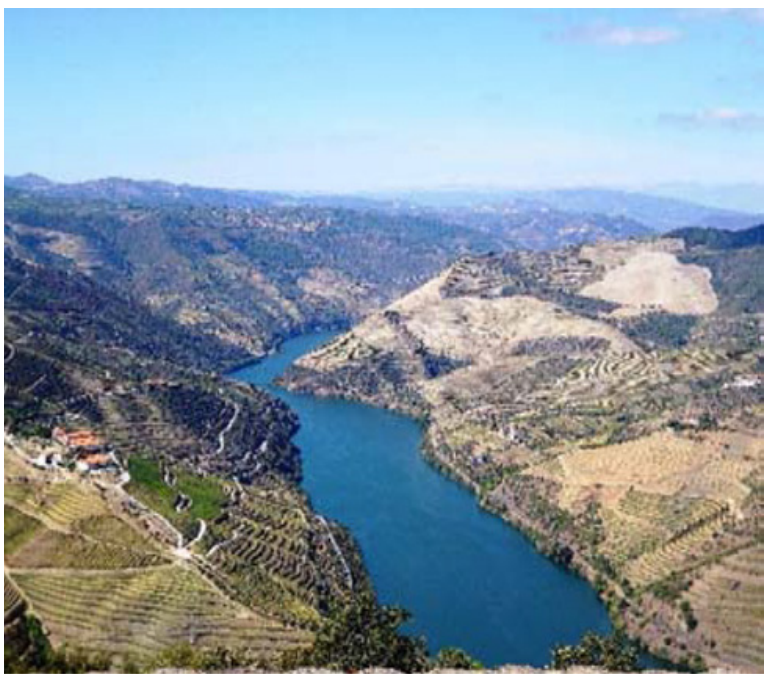

Figure 8. Paysage du Douro au Portugal.

exemple éminent de l'interaction de l'homme avec son environnement naturel.

\section{Portugal}

Région Viticole du Haut-Douro (Fig. 8)

Ce paysage est un des fleurons des paysages viticoles mondiaux. La région élabore du vin depuis quelque deux mille ans et sa principale production, le vin de Porto, est célèbre dans le monde entier depuis le XVIIIe siècle. Cette longue tradition a façonné un paysage culturel d'une beauté exceptionnelle qui reflète en même temps son évolution technique, sociale et économique. Ce paysage culturel impressionnant est toujours exploité avec profit par des vignerons respectueux des traditions. Les composants du paysage illustrent toute la palette des activités associées à la viticulture : terrasses, quintas, villages, chapelles, etc.

Ile volcanique du Pico (Fig. 9)

Ce site de 987 ha, est situé sur, la deuxième île de l'archipel des Açores par la taille. C'est un remarquable réseau de longs murs de pierre largement espacés, courant parallèlement à la côte et remontant vers l'intérieur de l'île. Ces murs ont été érigés pour protéger du vent et de l'eau de mer des milliers de petits enclos (currais) rectangulaires, 


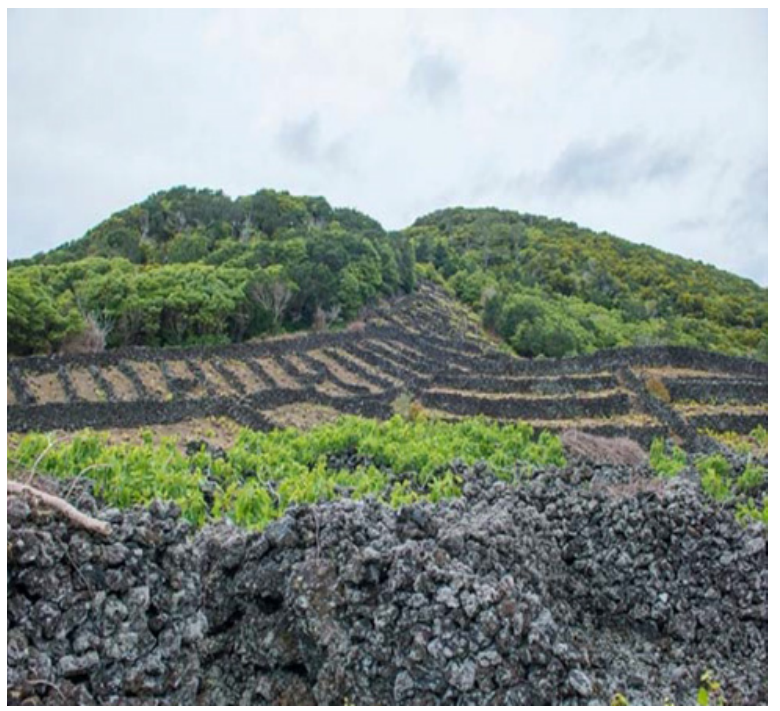

Figure 9. Ile de Pico au Portugal (c)Ko Hon Chiu Vincent/ Unesco).

accolés les uns aux autres. La présence de cette viticulture, dont les origines remontent au XVe siècle, est manifeste dans cet extraordinaire assemblage de petits champs, dans les maisons et les manoirs du début du XIXe siècle, ainsi que dans les caves, les églises et les ports. Ce paysage modelé par l'homme, d'une beauté extraordinaire, est le meilleur témoignage qui subsiste d'une pratique autrefois beaucoup plus répandue.

\section{Autriche}

Le paysage culturel de La Wachau (Fig. 10)

La zone classée est une partie de la vallée du Danube, entre Melk et Krems, dont le paysage conserve intactes de nombreuses traces de son évolution depuis les temps préhistoriques : traces architecturales (monastères, châteaux, ruines), urbanistiques (villes et villages) et liées à la culture de la vigne. L'architecture, l'habitat humain et l'utilisation agricole des terres de la Wachau illustrent de façon vivante un paysage essentiellement médiéval qui a évolué naturellement et harmonieusement à travers les âges.

\section{Autriche et Hongrie}

Le paysage culturel de Fertö et Neusiedlersee (Fig. 11)

A la frontière entre la Hongrie et l'Autriche, parmi tous les lacs salés du monde, cette zone est unique $\mathrm{du}$ point de vue de la relation organique, plurielle, ancienne et cependant toujours d'actualité entre l'homme et son milieu, typique du lac et de sa société. L'aspect traditionnel, en partie rural, de l'architecture des villages autour du lac, la structure de ces villages, l'unité des bâtiments disposés de façon homogène autour des places et le long des rues et plusieurs palais des XVIIIe et XIXe siècles qui s'inscrivent dans ce cadre paysager, sont les éléments caractéristiques, façonnés par l'homme, du paysage culturel. La culture de la vigne, la production vinicole et l'exploitation des roseaux, des activités séculaires, contribuent à pérenniser l'occupation des terres et l'utilisation de matériaux de construction traditionnels.

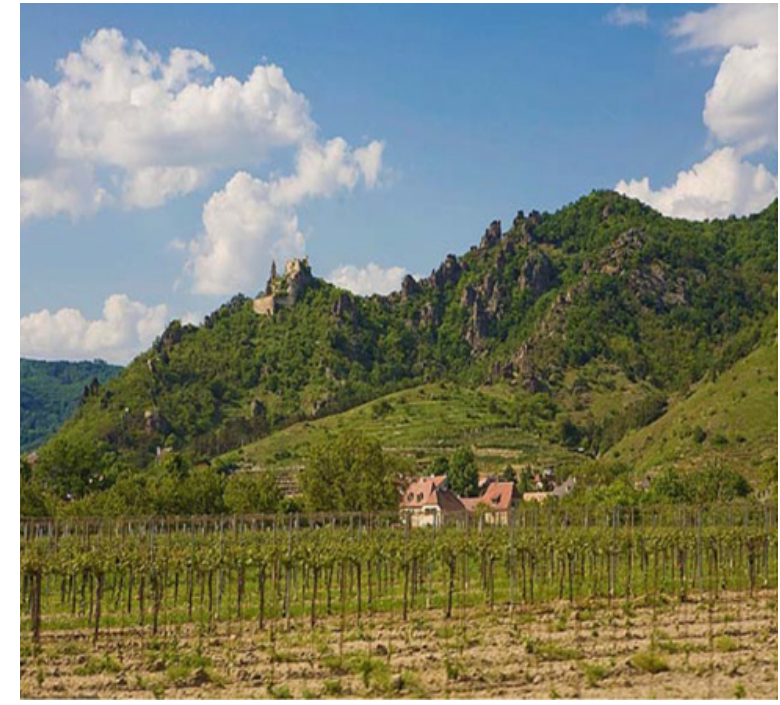

Figure 10. Paysage de la région de Wachau en Autriche (c)Ko Hon Chiu Vincent/Unesco).

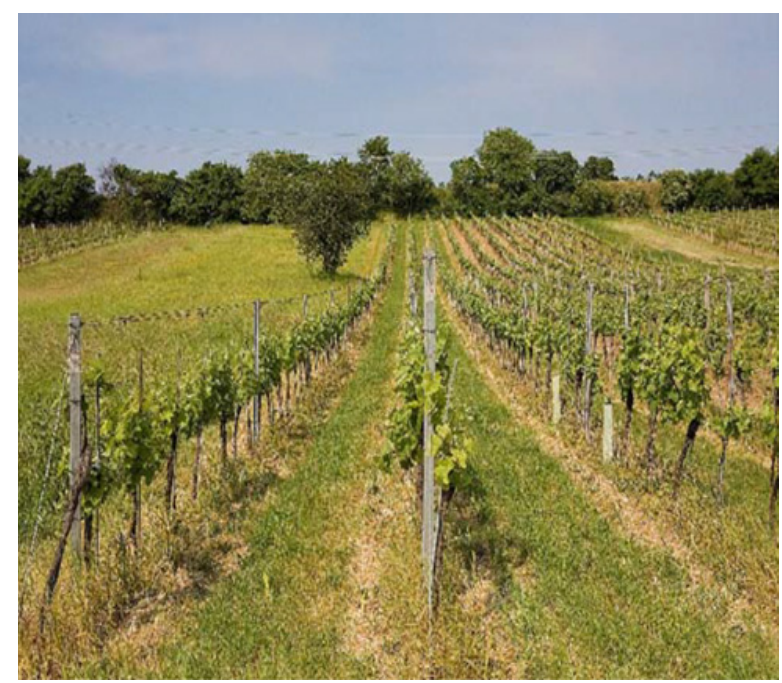

Figure 11. Paysage du site de Fertö et Neusiedlersee (C)Ko Hon Chiu Vincent/UNESCO).

\section{Allemagne}

La Vallée du Haut-Rhin moyen, également connue sous le nom de Rhin romantique (Fig. 12)

Le classement correspond à une section de 65 kilomètres de long de la vallée du Rhin entre Bingen et Coblence en Allemagne. Cette vallée, avec ses châteaux, ses villes historiques et ses vignobles, illustre de manière vivante la pérennité de l'implication humaine dans un paysage naturel spectaculaire et bigarré. Ce paysage est intimement lié à l'histoire et à la légende et exerce, depuis des siècles, une puissante influence sur les écrivains, les peintres et les compositeurs.

Les roches du site sont de type sédimentaire et ont été constituées à la période géologique du dévonien. La gorge fut creusée à des périodes géologiques plus récentes, bénéficiant de l'effondrement du fossé rhénan. Le fleuve coule dorénavant dans un canyon atteignant par endroit 200 mètres de profondeur, comme au niveau du rocher de la Lorelei. La vallée bénéficie d'un climat doux qui a permis de développer dans cette zone septentrionale un 


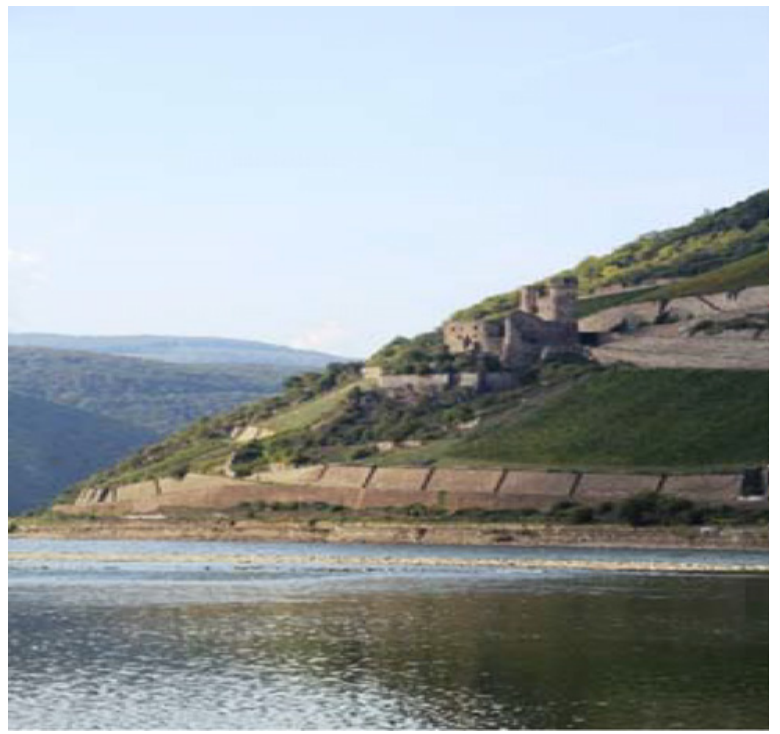

Figure 12. Paysage de la vallée du Rhin moyen en Allemagne.

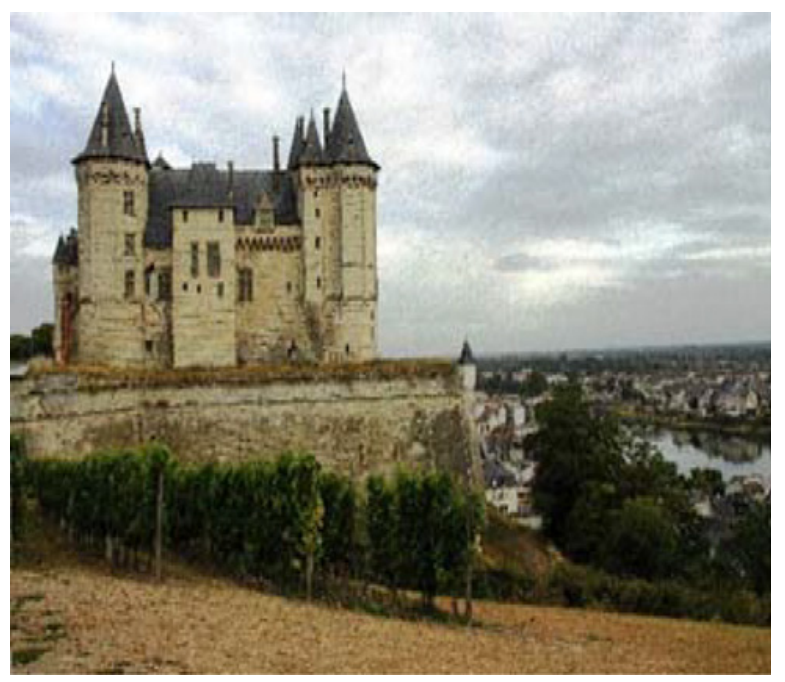

Figure 13. Paysage du Val de Loire.

vignoble aménagé en terrasses, majoritairement sur les versants exposés au sud.

\section{France}

Le Val de Loire entre Sully-sur-Loire et Chalonnes (Fig. 13)

C'est un paysage culturel exceptionnel, comprenant des villes et villages historiques, de grands monuments architecturaux, de nombreux châteaux, des terres agricoles et des vignes façonnées par des siècles d'interaction entre les populations et leur environnement physique. La vigne pousse sur des coteaux exposés au soleil, le long de la Loire et de ses affluents. Il existe une très grande diversité de sols et de paysages tout au long de la vallée.

La juridiction de Saint-Emilion (Fig. 14)

La viticulture a été introduite dans cette région fertile d'Aquitaine par les Romains et s'est intensifiée au Moyen Âge. Le territoire de Saint-Émilion a bénéficié de sa situation sur la route de pèlerinage vers SaintJacques-de-Compostelle et plusieurs églises, monastères et hospices y ont été construits à partir du XIe siècle. Le statut particulier de juridiction lui a été accordé

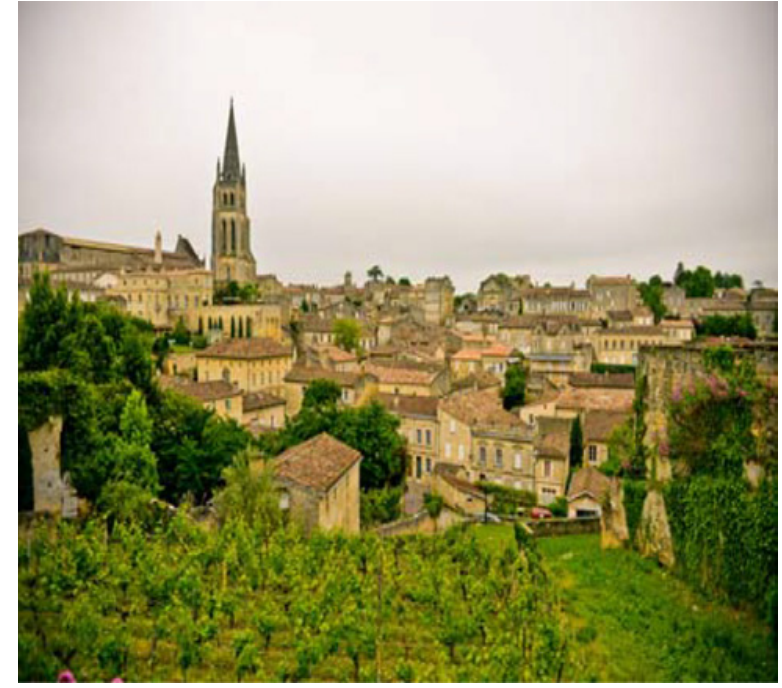

Figure 14. Paysage de Saint-Emilion (Source Nadiyka/Fotolia).

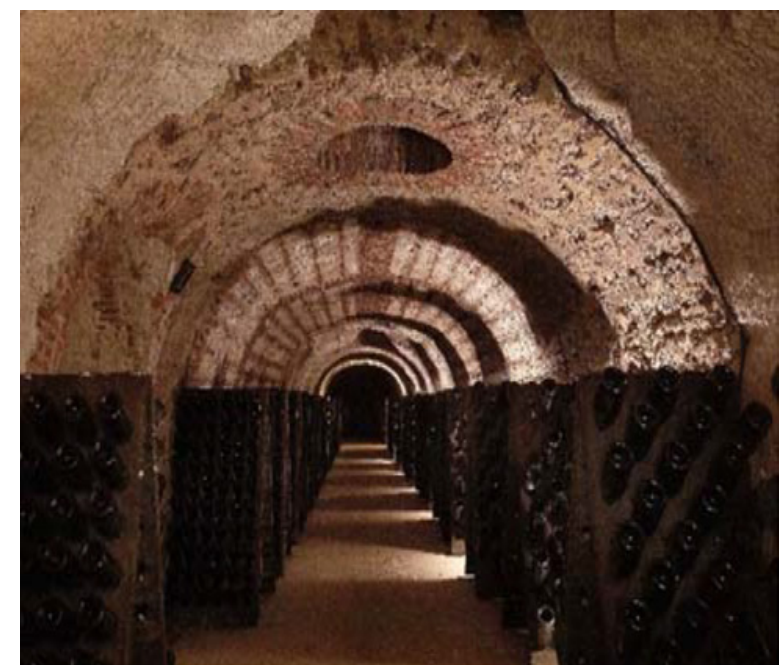

Figure 15. Cave de l'avenue de Champagne représentative du patrimoine historique de cette région, Copyright association paysages du Champagne. Photo Michel Jolyot/Copyright association paysages du Champagne/UNESCO.

au cours de la période du gouvernement anglais au XIIe siècle. Le vignoble de Saint-Emilion a été façonné par des générations successives de vignerons. Ceux-ci ont su faire évoluer la Juridiction, tout en préservant les témoignages remarquables de l'Histoire. Saint-Emilion déploie un patrimoine architectural spectaculaire, avec la cité médiévale et les églises romanes. Cet héritage du passé est également composé de grottes pittoresques, de moulins ou de pigeonniers que découvre le promeneur au bord des chemins.

\section{La Champagne (Fig. 15)}

Le site se compose de trois ensembles distincts : les vignobles historiques d'Hautvillers, Aÿ et Mareuilsur-Aÿ, la colline Saint-Nicaise à Reims ainsi que l'avenue de Champagne et le Fort Chabrol à Épernay. Ces trois ensembles, soit le bassin d'approvisionnement que forment les coteaux historiques, les unités de production (les caves souterraines) et les espaces de commercialisation (les maisons de Champagne), reflètent la totalité du processus de production de champagne. Les coteaux, maisons et caves de Champagne sont des 


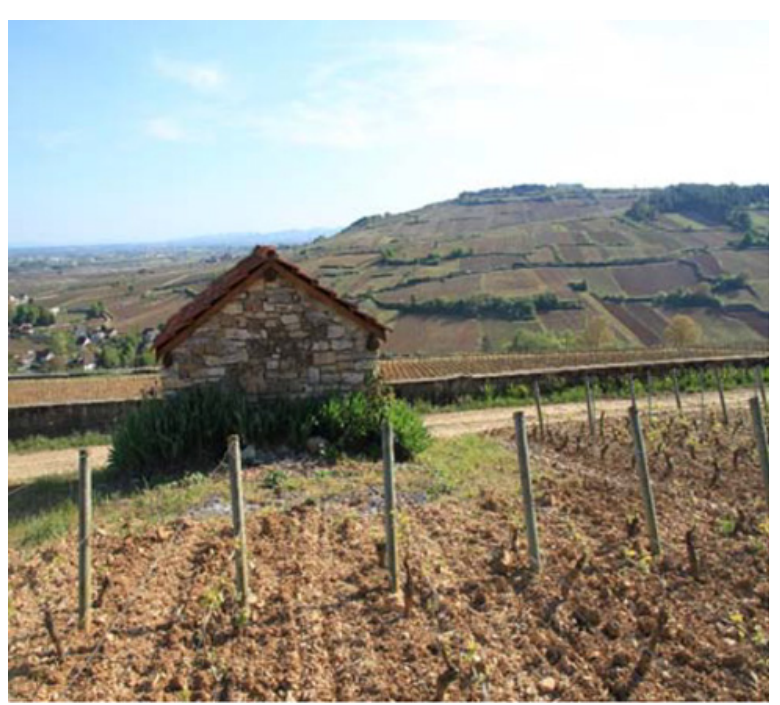

Figure 16. Paysage des climats de Bourgogne.

lieux où fut développée la méthode d'élaboration des vins effervescents, grâce à la seconde fermentation en bouteille, depuis ses débuts au XVIIe siècle jusqu'à son industrialisation précoce au XIXe.

Les coteaux, maisons et caves de Champagne, en particulier la colline Saint-Nicaise, avec ses carrières-caves monumentales et ses anciennes maisons de Champagne, et l'avenue de Champagne, avec les espaces de représentation des maisons de commerce, traduisent d'une manière exceptionnelle l'image symbolique unique au monde du champagne en tant que symbole de l'art de vivre à la française.

Les climats du vignoble de Bourgogne (Fig. 16)

C'est un ensemble de parcelles de vignes précisément délimitées sur les pentes de la côte de Nuits et de Beaune. Elles se distinguent les unes des autres par leurs conditions naturelles spécifiques (géologie, exposition, cépage...) qui ont été façonnées par le travail humain et peu à peu identifiées par rapport au vin qu'elles produisent. Ce paysage culturel est composé de plusieurs éléments : des parcelles viticoles et les unités de production associées, des villages et la ville de Beaune. Le site est un exemple remarquable de production viticole développé depuis le haut Moyen Âge avec une forte dynamique monastique.

\section{Conclusion}

De la diversité des reliefs et des climats naît une variété de paysages. L'esthétique de la vigne témoigne de cette subtile harmonie que l'homme a su établir avec la nature. Les paysages vignerons témoignent d'une diversité géologique unique et d'une histoire culturelle de la vigne et du vin sans égal.

Cette dimension, qui relie la terre à l'esprit, associe en premier lieu le savoir-faire du vigneron architecte et jardinier du terroir. Sa maîtrise technique est le fruit d'observations empiriques acquises de générations en générations et d'un esprit d'innovation permanent. L'ingéniosité de l'homme ne s'arrête pas à la vigne. L'architecture des chais, des caves mais également celle des villages, des églises, ou d'autres bâtisses historiques participe pleinement à l'harmonie et à la splendeur des paysages viticoles mondiaux.
Le label Réseau International Paysages Viticoles établi à partir de la Charte de Fontevraud témoigne de la qualité des démarches volontaires et concertées sur des terroirs viticoles, où tous les acteurs du territoire (collectivités locales, syndicats de producteurs, opérateurs économiques, culturels et touristiques, universités et laboratoires) sont engagés, à partir d'un diagnostic paysager et un plan d'action. L'objectif de cette démarche est d'optimiser la production viticole ainsi que la gestion paysagère et environnementale, avec des offres culturelles et touristiques innovantes dans une logique de développement durable (Fig. 3).

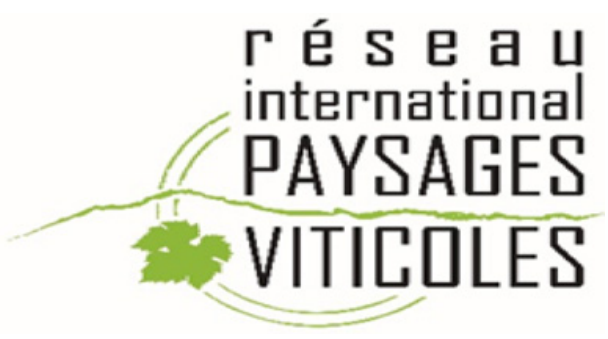

Figure 17. Logo du Réseau International Paysage Viticole établi sur la base de la charte de Fontevraud. http://www . vignevin.com/recherche/territoires/paysagesviticoles/reseau-international-paysagesviticoles.html

Le professionnel de la vigne, par son influence sur l'espace, est aussi un professionnel du paysage qui n'a pas pour autant conscience de la valeur esthétique et patrimoniale de son terroir. À l'image d'une dégustation, son paysage est l'expression conjuguée du plaisir des sens ; il doit apprendre à le connaître et le faire apprécier.

Vitrines des pratiques viticoles, les paysages sont aujourd'hui porteurs d'enjeux écologiques (bonnes pratiques environnementales, maintien de la qualité des eaux, contribution à la conservation des sols, valorisation d'une biodiversité «fonctionnelle et écologique »). Ils ont également une valeur économique pour l'exploitation et la région (image promotionnelle du vin, tourisme, foncier), sociale (cadre de vie, identité locale, médiation), culturelle et patrimoniale (rites, pratiques, sentiers, paysage et architecture). Ce sont des liens identitaires forts, supports de projets collectifs qui placent les vignerons au cœur du développement durable de leur territoire. Le paysage viticole présente l'intérêt de fédérer la profession autour d'un sujet identitaire qui doit associer les collectivités locales et l'ensemble des acteurs du territoire. C'est la base de la gouvernance patrimoniale mais également écologique des terroirs.

Mais, cet écosystème culturel est fragile. La modernité, dans son approche fonctionnelle a souvent occulté ces richesses patrimoniales léguées par les anciens, héritage parfois perçu comme une contrainte face aux évolutions technologiques et aux impératifs économiques. Sachons protéger et transmettre à nos enfants ce patrimoine esthétique, mémoire d'intelligence, de sensibilité, de sueur, et parfois de combats. C'est un capital qu'il convient de connaître, de préserver et de valoriser, si l'on veut pérenniser la culture du vin. 
L'inscription au patrimoine mondial de l'Unesco contribue à une très forte dynamique collective interdisciplinaire qui associe l'ensemble des acteurs d'un territoire et permet d'initier une réflexion prospective sur ses atouts et faiblesses afin de mettre en œuvre des plans d'action adaptée à chaque contexte. Néanmoins toutes les régions viticoles ne pourront pas accéder à ce label, graal de la reconnaissance patrimoniale. Au-delà de la complexité du montage, de nombreuses régions dotées d'une valeur esthétique remarquable, en particulier dans les pays du Nouveau Monde, peuvent difficilement s'intégrer dans l'approche « patrimoine mondial » Unesco. Peut-être est-il utile de réfléchir à une forme de reconnaissance internationale qui pourrait notamment associer l'OIV, en liaison avec d'autres organisations internationales liées au patrimoine ou à l'écologie, sur la base d'une gouvernance des paysages en lien avec les autres composantes des terroirs, en particulier la biodiversité, en adéquation avec les définitions du terroir et de la viti-viniculture établies par l'OIV. Cette approche permettrait de mobiliser la plupart des régions viticoles du monde autour d'un projet collectif et interdisciplinaire, de nature à valoriser l'image globale de la filière viticole. Les travaux menés dans le cadre du Réseau International Paysages Viticoles, en lien avec la charte de Fontevraud, qui intègre une gouvernance globale, pourrait servir de support pour une telle approche.

\section{Références}

[1] J. Rochard, A. Lasnier, Application aux secteurs viticoles de la Convention européenne sur les paysages, revue des œnologues, 121 (2006)

[2] C. HERBIN, J. ROCHARD, Les paysages viticoles, éditions Féret (2005)

[3] J. ROCHARD, Traité de viticulture et d'œnologie durable, éditions Avenir œnologie (2005)

[4] UNESCO, Convention concernant la protection du patrimoine mondial culturel et naturel, Unesco. http://whc.unesco.org/ (1972)

[5] P.M. TRICAUD, L'application aux paysages de vignobles des critères d'éligibilité au patrimoine mondial. ICOMOS. www . icomos . org (2001)

[6] UNESCO, Établir une proposition d'inscription au patrimoine mondial, Unesco. http://whc.unesco.org/ (2011)

[7] UNESCO, Gérer le patrimoine mondial naturel, Unesco. http://whc.unesco.org/ (2012)

[8] UNESCO, Gérer le patrimoine mondial culturel, Unesco. http://whc. unesco.org/ (2014)

[9] OIV, guide OIV pour une vitiviniculture durable : production, transformation et conditionnement des produits, OIV-CST 01-2008. www . oiv . int (2008)

[10] OIV, Définition du «terroir » vitivinicole : résolution OIV/VITI 333/2010. www . oiv . int (2010) 